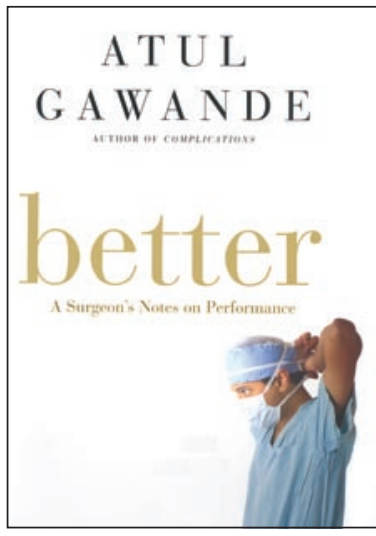

\title{
Better
}

\section{A surgeon's notes on performance}

\author{
Atul Gawande \\ Metropolitan Books. New York, New York, USA. 2007. \\ 288 pp. \$24.00. ISBN: 978-0-8050-8211-1 (hardcover).
}

\section{Reviewed by Ara Darzi and Oliver Warren}

Department of Biosurgery and Surgical Technology, St. Mary's Hospital, Imperial College London, London, United Kingdom.

E-mail: a.darzi@imperial.ac.uk

I n Better: a surgeon's notes on performance, Atul Gawande, best-selling author of Complications: a surgeon's notes on an imperfect science, general and endocrine surgeon at Brigham and Women's Hospital, and assistant professor at Harvard Medical School and Harvard School of Public Health, investigates human performance in medicine. He takes the reader to courtrooms, clinics, war zones, and execution chambers to demonstrate the incredible environments in which doctors care for and treat patients. While drawing on a vast array of sources, including his own personal experience, Gawande interweaves into nearly every chapter fascinating vignettes about the most important person in his view of medicine: the patient. By ensuring that every message is personalized in this manner, he brings a warm, humane touch to this superb book.

Gawande defines "three core requirements" for successful performance in medicine - diligence, doing right, and ingenuity - and in turn considers specific problem areas in each. He cites three distinct examples in which diligence is essential to the successful execution of medical therapy: the WHO's mop-up of a polio outbreak in rural India; the efforts of the US military's medical staff to save the lives of servicemen seriously injured in Iraq; and the work of infection-control specialists to ensure hand washing to prevent the spread of hospitalacquired infections. The numbers cited in each example are impressive and daunting: 4.2 million Indian children vaccinated by 37,000 vaccinators over three days; 60 to 70 surgeons supporting the 130,000 troops fighting in Iraq (where injuries are horrific); 2 million Americans contracting hospital-acquired infections per annum. All of these efforts are demonstrably linked by the almost obsessive diligence of the clinicians responsible and their need to "make a science out of performance."

When considering "doing right," the author tackles a number of compelling issues, including the litigious nature of American society toward health care providers. We are introduced to two interesting individuals, a physician colleague of Gawande's whose son was harmed by medical error and an ex-clinician now practicing as a medical malpractice lawyer, and thus gain novel insight into the difficulties associated with rectifying the occasional harm that may inevitably result from health care. Gawande's approach is novel and intelligent and allows all parties to consider this troublesome area from enlightened viewpoints. As he declares, litigation is a "singularly unsatisfactory solution," bringing out the worst in everyone and often failing to help those injured by medical error. The search continues for more productive ways of recompensing victims, but creative potential solutions are offered and are worthy of consideration.

Gawande moves on to consider what may be the most controversial topic in the book: the role of physicians in state-sanctioned executions. "Doctor D" is an anti-capital punishment activist who has supervised the executions of six of his "patients" and who donates his entire fee for this service to a children's shelter. He considers those on death row to be "legally" terminally ill and thus feels a responsibility to ensure their final minutes are as pain free as possible. The paradox is intriguing, and Gawande is not judgmental. His moral indignation at the very thought of colleagues participating in this process rescinds to a realization that the ethical issues are perhaps more complex than first thought, a realization we share as readers.
Finally "ingenuity" is addressed, a matter "more about character and less about superior intelligence." The near-obsessive nature required not only to attain excellence, but to then perpetually evolve to stay ahead is illustrated by the fascinating tale of LeRoy Matthews and Warren Warwick, pediatricians who led the development of centers of expertise for cystic fibrosis. As fellow clinicians, we find these stories describing phenomenal improvements in patients' results through the targeted efforts of a few doctors to be inspirational and to serve as examples of what can be achieved if a culture of intellectually rigorous self-improvement is adopted within a unit. However, the next logical step is to question why we are not all this way. Gawande does not shrink from considering this bell curve of clinician performance and how it applies to his surgical practice and medicine as a whole. We are in an uncomfortable period for clinicians, as more and more of our work is subject to public scrutiny and objective assessment, a situation that meets with significant opposition from many colleagues. How, when, and by whom should this grading occur? Will being in the below-average group be used against us? Will those at the bottom be remunerated less than those at the top? Answers are not offered to all these questions, but as fellow surgeons, we share the author's only definitive conclusion - that there is no shame in being average, but settling for average is unacceptable.

Better opens a door to the oft-closed world of medical performance for careful scrutiny, explores variations in standards of clinical practice, and challenges all involved in health care to adopt the role of "positive deviant." By refusing to flinch when faced with controversy, Gawande has created an essential text for both patients and their doctors, to the betterment of us all. 